\section{Contribución de la formación literaria a la educación integral: análisis del currículo básico de Educación Primaria} Contribution of literary training to integral education: analysis of the basic curriculum of Primary Education

\author{
ELIA SANELEUTERIO \\ Departament de Didàctica de Llengua i la Literaura. Universitat de València. \\ (D) https://orcid.org/0000-0003-4060-9578 \\ ROCÍO LÓPEZ-GARCÍA-TORRES \\ Departamento de Ciencias de la Educación. Universidad CEU Cardenal Herrera \\ (D) https://orcid.org/0000-0001-5647-764X
}

\section{RESUMEN}

El proceso educativo conlleva siempre una relación con el valor: en esencia y fundamento resulta valioso. Al venir enmarcado por contextos legales, podemos afirmar que el Real Decreto $126 / 2014$, por ser educativo, se asienta en valores, unos u otros. Para esta investigación se ha llevado a cabo un análisis del contenido axiológico del bloque de dicho currículo básico dedicado a la educación literaria. Para ello se parte de un planteamiento integral de educación al que la literatura contribuye con un papel fundamental. El objetivo del estudio es demostrar hasta qué punto esto se ve reflejado en el texto legal de referencia, estimulando la reflexión sobre elementos que puedan suponer una mejora en próximas acciones normativas, incluida la potenciación del papel de la Literatura Infantil y Juvenil (LIJ) en este proceso.

\section{ABSTRACT}

The educational process always involves a relationship with value: in essence and foundation it is valuable. Always framed by legal contexts, it is possible to say that, since it is educational, the Royal Decree $126 / 2014$ is based on values. In this research an analysis of the axiological content of this basic curriculum is carried out, concretely the block dedicated to literary education. The study starts from an integral approach of education to which literature contributes with a fundamental paper. The objective is to demonstrate how this is represented in the referenced legal text, stimulating the consideration of elements that may lead to an improvement in future normative actions, including the strengthening of the role of the children's and youth literature in this process.

Para citar este artículo: Saneleuterio, E. y López-García-Torres, R. (2019). Contribución de la formación literaria a la educación integral: análisis del currículo básico de Educación Primaria. EA, Escuela Abierta, 22, 29-38. doi:10.29257/EA22.2019.04
Recibido: 02/02/19

Aceptado: 29/07/19

PALABRAS CLAVES Currículo básico, análisis de contenido, educación integral, axiología, educación literaria.

\section{KEYWORDS}

Basic Curriculum, content Analysis, Comprehensive Education, Axiology, Literary Education. 


\section{INTRODUCCIÓN Y PROPÓSITOS DE LA INVESTIGACIÓN}

Es sabido que la educación conlleva siempre una relación con el valor; es más, la educación en su misma esencia y fundamento es valiosa. Sabemos, además, que el proceso educativo no se da en el vacío, sino que tiene lugar siempre enmarcado en un contexto legal.

La legislación educativa se asienta, pues, en unos principios y valores que las instituciones escolares asumen y expresan en las diferentes dimensiones de su planificación. Y es que sabemos que los valores impregnan el hecho educativo, lo orientan y están presentes en el propio proceso. Es en este sentido en el que entendemos que resulta imposible elaborar una norma sobre educación sin un fundamento axiológico.

Así, pues, el Real Decreto 126/2014, de 28 de febrero, por el que se establece el currículo básico de la Educación Primaria, por ser educativo, se asienta necesariamente en valores.

Para nuestra investigación hemos llevado a cabo un análisis del contenido axiológico del currículo básico de Lengua Castellana y Literatura, concretamente del bloque 5, dedicado a la educación literaria (MECD, 2014, p. 19385). La razón es que apostamos, desde la reflexión crítica, por la contribución de la literatura a la educación integral.

El Bloque 5, Educación Literaria, asume el objetivo de hacer de los alumnos y alumnas lectores cultos y competentes, implicados en un proceso de formación lectora que continúe a lo largo de toda la vida. Para eso es necesario alternar la lectura, comprensión e interpretación de obras literarias cercanas a sus gustos personales y a su madurez cognitiva con la de textos literarios y obras completas que aporten el conocimiento básico sobre algunas obras representativas de nuestra literatura.

La información y la interpretación de textos u obras no es unidireccional de profesor a alumno, sino que es este último el que debe ir adquiriendo, con la guía del docente, los recursos personales propios de un lector activo, capaz de ver en la obra literaria una ventana abierta a la realidad y a la fantasía y un espejo en que el que tomar conciencia de sí mismo y del mundo que le rodea. Del mismo modo, esa toma de conciencia del mundo y de uno mismo se ve favorecida por la actividad lúdica y creativa del alumnado en la producción de textos personales de intención literaria. (MECD, 2014, p. 19380)

Como mostramos a continuación, si bien la materia de Lengua Castellana y Literatura se convierte en un instrumento eficaz de transmisión de valores intelectuales, sociales, estéticos, instrumentales... e individuales, cosa que se pone de manifiesto por la atención prestada - en diferente medida - a estos valores, el bloque dedicado a la educación literaria supondría escasa o nula consideración hacia los liberadores, temporales, morales, afectivos, espaciales, corporales, ecológicos o religiosos. Ello plantea la necesidad de propuestas educativas para contribuir, desde la literatura, a la formación integral de los educandos a través del currículo en Educación Primaria.

La inclusión de la literatura en los programas educativos siempre ha sido una fuente potenciadora de ejes transversales del currículum e incluso de la formación de la propia identidad (Aparicio, 2016). Yendo más allá, si la educación literaria es un punto clave en la contribución normativa a la educación integral, queda justificado el objetivo principal del presente estudio: nos proponemos demostrar hasta qué punto y en qué distribución dicha representación se cumple en el texto legal vigente que regula los contenidos mínimos de educación literaria durante la etapa de Primaria, centrándonos en el bloque 5 de Lengua Castellana y Literatura. 


\section{MARCO TEÓRICO}

En la actualidad es ampliamente admitido que el fin último de la educación es el desarrollo integral de la persona. "La expresión educación integral, aunque puede interpretarse en sentidos muy variados, todos ellos incluyen el concepto de totalidad: la educación del hombre completo, de todas y cada una de sus facultades y dimensiones" (Gervilla, 2000, p. 41). Observamos, pues, que la educación integral se encuentra básicamente vinculada a la esencia de la persona, sujeto de la educación.

Nuestro modelo axiológico de educación integral asume la definición de persona del profesor Gervilla (2008, p. 64): "Animal de inteligencia emocional, singular y libre en sus decisiones, de naturaleza abierta o relacional, en el espacio y en el tiempo". Esta concepción de persona y los valores que es capaz de generar constituyen una estructuración categorial que nos permite detectar la presencia o ausencia de ciertos valores en cualquier modelo educativo, proyecto, reglamento de centro o ley, así como la importancia otorgada a cada una de las categorías.

Uno de los valores más desatendidos en los planes educativos son los estéticos, que suelen concentrarse casi exclusivamente en las áreas artísticas o en la educación literaria. Precisamente una de las subcategorías incluidas en los valores estéticos serían los literarios, cuya atención a menudo se halla vinculada al potenciamiento simultáneo de otros valores que conformarían el modelo de educación integral referido (Fraga, 2005; Tejerina y Echevarría, 2007). Si nos damos cuenta, la literatura infantil y juvenil (LIJ) no solo está vinculada a la lengua y a la literatura:

[...] además está relacionada con otras áreas como Conocimiento del Medio, Educación Artística, Matemáticas, Formación Religiosa, Ética o, incluso, con Educación Física. Son, pues, obras que tienen una finalidad educativa evidente, o, al menos de este modo se intentan presentar y obras que al mismo tiempo deben incluir unos valores literarios de los que en ocasiones carecen. (Llorens, 2000, p. 1)

No en pocas ocasiones se ha hablado de la literatura y sus valores como fuente importante de la educación, capaz de desarrollar en los niños y jóvenes actitudes ante la vida. Es cierto que el cuento tiene un valor educativo (Martí, 2004; Huertas, 2006) que puede ser aprovechado legítimamente, pero no debemos nunca olvidar su función principal: convertirse en fuente de placer estético para el lector.

No se pueden desatender, en la elección de obras literarias para la escuela, los valores literarios que en ellas deberían contenerse; es decir, la presencia en un libro infantil de ciertos valores no justifica per se que el docente haga pasar como literarios textos que no lo son, tal y como vienen advirtiendo los especialistas desde hace décadas (Cerrillo, 1996; Sánchez Corral, 1995). Rodríguez y Gutiérrez (2013) van incluso más allá e insisten en el peligro de la instrumentalización de la obra literaria.

Los valores literarios pueden definirse como "las conjugaciones del aspecto estético con el lingüístico" (Saneleuterio y López-García-Torres, 2017, p. 344). Sobre ellos, junto con sus facetas social y ética, se asienta la educación literaria, que busca principalmente enseñar a apreciar el valor literario en un texto, es decir, encontrar "relaciones entre su forma y su contenido, tanto denotativo como connotativo, las cuales, además de ser capaces de emanar belleza, provocando experiencias de deleite, presentan la virtud de actualizarse de manera personalizada" (Saneleuterio y López-García-Torres, 2017, p. 344).

Una obra desprovista de lenguaje literario difícilmente puede transmitir los valores estéticos contenidos en el bloque 5 del currículo, cuya cuantificación evidencia el presente artículo. Sobre ello se ha investigado mucho (Cerrillo y Yubero, 1996; González y Nieto, 2001; Nieto y González, 2002; Etxaniz, 2008). Sin embargo, cabe hacer hincapié en que la sola presencia de valores estéticos en una obra literaria no garantiza una adecuada educación del gusto 
estético, pues la acción docente puede ir encaminada a hacer un uso abusivo de la misma, obviando los valores literarios de ciertas obras, incluso cuando pueden ser ampliamente reconocidos por la tradición y la crítica, en aras de un potenciamiento de ciertos valores también incluidos en ellas.

Finalmente, cabe señalar el papel fundamental que en todo ello juegan, más allá de la mera animación a la lectura, los proyectos pedagógicos encaminados al fomento del hábito lector (Cerrillo, 1996).

\section{METODOLOGÍA}

Pretendemos nuestra investigación desde la reflexión crítica, a través de un diseño de corte cualitativo, con tratamiento estadístico de datos, cuya validez viene controlada mediante juicio de expertos (López-García-Torres, 2012). Los resultados tuvieron lugar tras la aplicación de estadísticos elementales como son las frecuencias, porcentajes y ordenaciones mediante el programa informático Microsoft Excel.

El documento sobre el que hemos realizado nuestra investigación es el texto de los contenidos mínimos de la materia Lengua Castellana y Literatura, promulgados oficialmente mediante el Real Decreto 126/2014, de 28 de febrero, por el que se establece el currículo básico de la Educación Primaria, centrando nuestro foco de atención en el bloque 5, reservado a la educación literaria y que constituye, por tanto, nuestro corpus principal de análisis.

En concreto, los indicadores en que se fundamenta la interpretación son la ausencia o presencia de valores, y dentro de esta última, la frecuencia de aparición de estos.

La técnica utilizada en nuestra investigación es el análisis de contenido axiológico (Gervilla, 2008), utilizada en estudios analíticos amplios previos (López-García-Torres, 2012; Saneleuterio y López-García-Torres, 2017). Los valores han sido extraídos con base en un sistema de categorías, tal y como exige el análisis de contenido (Bardin, 2002), confeccionado a la luz del modelo axiológico de educación integral descrito arriba: se trata de los valores corporales, valores intelectuales, valores afectivos, valores individuales, valores liberadores, valores morales, valores sociales, valores ecológicos, valores estéticos, valores religiosos, valores instrumentales, valores espaciales y valores temporales, a los que añadimos los globalizadores.

Tal y como los definimos en López-García-Torres y Saneleuterio (2019), los valores corporales estarían especialmente vinculados con la dimensión física de la persona o con el movimiento y la actividad corporal. Por su parte, los valores intelectuales serían los que relacionan directamente con la naturaleza y facultad racional del ser humano: la actividad intelectual y los procesos mentales, sus conocimientos y sus habilidades cognitivas. Los valores afectivos se relacionan directamente con la dimensión afectiva de las personas, es decir con sus sentimientos, emociones y pasiones. Respecto a los valores individuales, se refieren principalmente al aspecto particular e íntimo de la persona, sus peculiaridades e identidad personal, mientras que los liberadores serían aquellos valores relacionados con la capacidad de libre elección de las personas y la toma de decisiones, así como con las acciones encaminadas a alcanzar sus deseos y pretensiones. Los valores morales toman como punto de referencia central los fines y acciones relacionados con el bien en cuanto tal, acorde con la dignidad humana y lo éticamente bueno: el deber y las normas morales que rigen la conducta humana. Se diferencian de los valores sociales en la medida en que estos son relativos principalmente a la sociedad, su organización y las relaciones interpersonales e institucionales. Si continuamos con los valores ecológicos, los identificamos por estar directamente relacionados con la naturaleza, sus propiedades y los seres vivos, así como con las relaciones que las personas establecen con el 
medio ambiente: su consideración, disfrute, cuidado y defensa. Los valores estéticos quedarían ligados a aquellas creaciones del ser humano y las manifestaciones de la naturaleza que son deseadas o deseables por su belleza. Los valores religiosos, por su parte, serían aquellos valores relativos al sentido religioso y trascendente de la vida, las creencias religiosas y las prácticas relacionadas con la divinidad. Los valores instrumentales tienen que ver con los instrumentos y recursos, materiales, humanos y procedimentales, que estimamos como medios para conseguir fines deseados. Los valores espaciales y temporales son aquellos valores que se refieren, respectivamente, al tiempo o al lugar como algo positivo y deseable. Finalmente, se hace necesario incluir en la investigación una categoría de valores globalizadores (López-García-Torres, 2012) para aquellos valores con gran amplitud semántica.

\section{RESULTADOS}

En la Figura 1 presentamos los resultados del análisis descriptivo explicitado en el apartado anterior, consecuencia del cual descubrimos los valores que encierra el bloque 5, así como su importancia o jerarquía, desde la perspectiva de la educación integral sostenida.

\section{Figura 1}

Jerarquía axiológica "Bloque 5. Educación Literaria". Elaboración propia

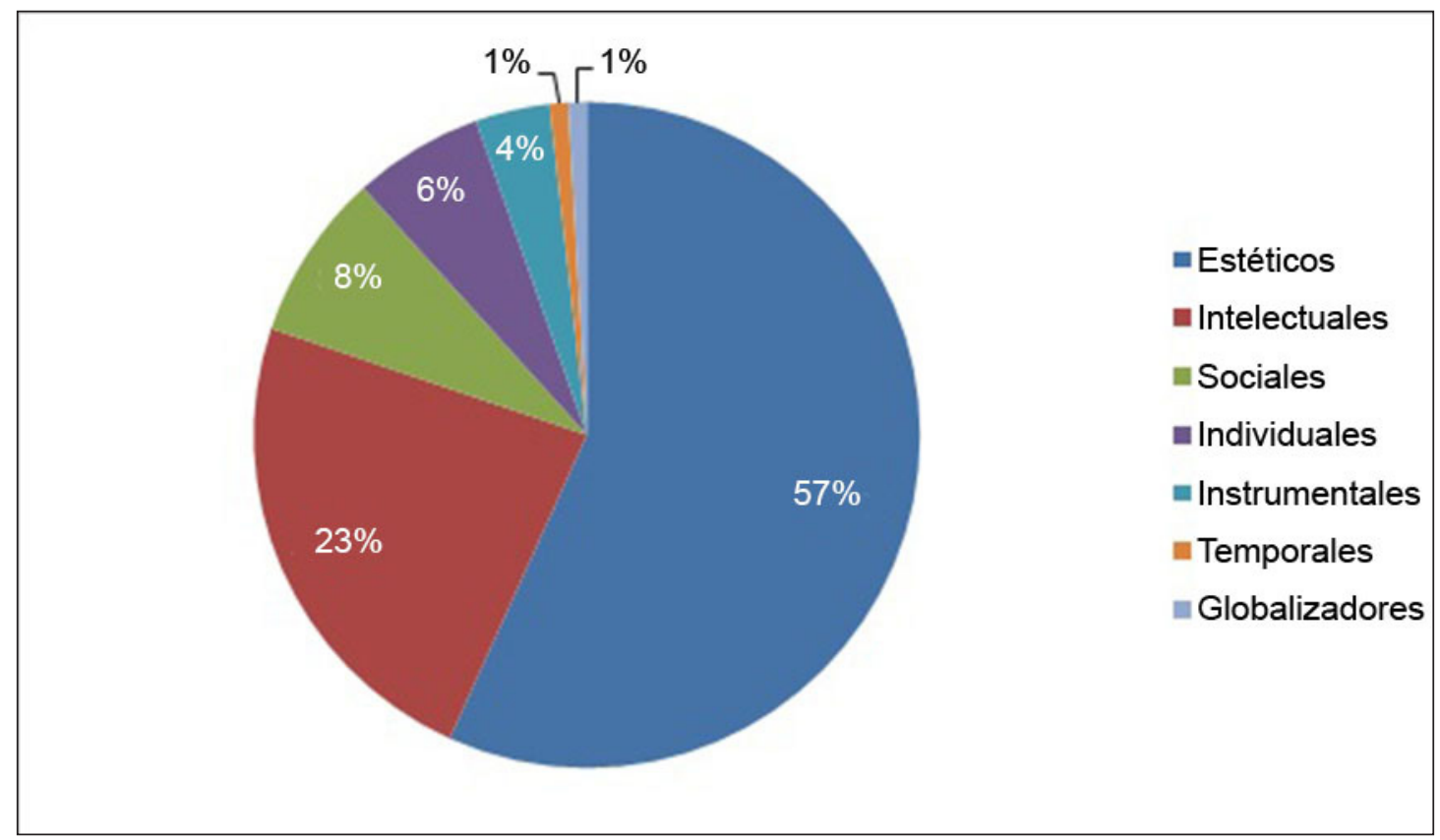


En primer lugar, comprobamos como solo quedan reflejados la mitad de valores categorizados. Es decir, no están representados en ninguna medida los valores corporales, afectivos, liberadores, morales, ecológicos, religiosos o espaciales.

Asimismo, el resultado más evidente de nuestro análisis es la fuerte tendencia centralizadora de los valores estéticos, que alcanzan casi un 60 \% de representación sobre el total de valores de ese bloque.

Los seis restantes se reparten en proporciones también llamativas. Podemos observar que casi un cuarto de la representación total lo ocuparían los valores intelectuales.

Y respecto al $20 \%$ restante, destaca la presencia de los valores sociales, individuales e instrumentales, por este orden, mientras que los temporales y globalizadores apenas cuentan con la representación mínima - que corresponde con una sola unidad registrada-.

\section{DISCUSIÓN}

Hemos señalado en otro lugar (López-García-Torres y Saneleuterio, 2015; Saneleuterio y López-García-Torres, 2017) la escasa representación de los valores estéticos y, en concreto, de los literarios en las leyes educativas. Dado que estas son las que regulan el proceso educativo en cada uno de sus momentos de concreción, consideramos que la mayor presencia de los valores estéticos en la educación literaria es garantía de que estos estén atendidos en la formación, si bien hemos insistido al mismo tiempo en que no deberían ser exclusivos de ella, sino que convendría contemplarlos también transversalmente en mayor medida.

En los últimos años, se están produciendo fenómenos de reivindicación en esta línea incluso documentados por la prensa, hecho que tiene mayor impacto en la sociedad general que las publicaciones académicas, que por supuesto también proliferan. Docentes y especialistas en educación entienden como imprescindible la presencia de lo artístico en los planes educativos.

Defienden que la formación artística es básica para un crecimiento integral del alumnado y necesaria para la adquisición de las competencias básicas, y abogan por que la Música y la Plástica tengan el mismo tratamiento que las otras materias prácticas, permitiendo reducir ratios mediante desdoblamientos o agrupamientos flexibles. (M. F. R., 2015)

Por otro lado, aunque el hecho de que el documento legal analizado no mencione explícitamente un valor no implica que este no pueda ser atendido en la práctica docente, no podemos negar que la ausencia de siete tipologías de valores en el bloque de contenidos mínimos de la educación literaria (Figura 1) resulta un punto destacable para su discusión, no solo porque suponen el 50 \% de los valores categorizados, sino también si consideramos la naturaleza de estos: los valores liberadores o afectivos, por ejemplo - aunque también los morales, corporales, ecológicos, etc.-, se han relacionado tradicionalmente con la LIJ en su función social y formativa (Bassa i Martín, 2003-2004; Borda Crespo, 2000); sin embargo, según los resultados de nuestro análisis axiológico mostrados en la Figura 1, su atención no quedaría prevista en el currículo de contenidos mínimos para la Educación Primaria.

$\mathrm{Al}$ respecto afirma Simone Sousa (2008): "La literatura infantil desde siempre ha sido un campo donde se han sembrado valores que, sin duda, han formado parte de la mentalidad colectiva. Los valores, sean tradicionales o nuevos, determinan la temática y las peculiaridades formales que diferencian las literaturas infantiles de ayer y de 
hoy". En efecto, el factor pedagógico-formativo de la literatura infantil es innegable y, en la mayoría de las ocasiones, es infrecuente abordar la lectura en el aula sin comentar numerosas cuestiones que afectan o se relacionan con la jerarquía axiológica. Dicho de otro modo, aunque no todos los valores categorizados aparezcan explícitamente en el documento estudiado, en la práctica docente es indispensable su consideración si queremos lograr la plena comprensión de los textos, y esto no debería dejarse al libre arbitrio de maestros y maestras, especialmente porque este objetivo se orienta al mismo tiempo hacia la educación integral.

En cuanto a los valores intelectuales, nuestro estudio muestra con contundencia que son los segundos más dominantes en el bloque. Sin embargo, el hecho de que representen solo el $23 \%$ resulta muy revelador, sobre todo si lo comparamos con la gran hegemonía que estos valores suelen suponer en las distribuciones axiológicas de textos normativos, cuando estos se analizan en su conjunto (López García-Torres, 2012).

A pesar de ello, y teniendo en cuenta la distribución axiológica del resto de valores, consideramos que esta representación les confiere incluso demasiada importancia, pues sería reflejo de que la redacción del texto legal estaría primando todavía la productividad académica o la preeminencia del éxito escolar entendido de manera parcial, no por encima de los valores estéticos, afortunadamente, pero sí de otros que merecerían mayor atención en su relación con la educación literaria. Nos referimos, principalmente, a los sociales y a los individuales, que podrían contar con mayor representación a costa de los intelectuales, así como a los que directamente están ausentes en el corpus analizado.

\section{CONCLUSIÓN: CONTRIBUCIONES Y SIGNIFICACIÓN CIENTÍFICA DEL ANÁLISIS}

El fundamento axiológico mostrado corresponde a los valores que encierra el currículo básico de Lengua Castellana y Literatura en su bloque 5 de contenidos. Una representación más proporcionada de valores, aunque pudiera parecer más equitativa como contribución a la educación integral, en realidad sería contraproducente, pues se ha de contemplar la distribución axiológica de la formación en su conjunto.

Así pues, con este estudio concluimos que, en la representación axiológica del bloque de contenidos mínimos analizado, correspondiente a la literatura que se estudia en Primaria, prima la educación estética. Ello lo valoramos como adecuado porque esta es poco atendida en el resto del currículo, incluso en el resto de bloques de la propia asignatura de Lengua Castellana y Literatura.

Por otro lado, consideramos como punto débil del Real Decreto la ausencia, en el bloque de contenidos de educación literaria, de siete tipologías de valores, principalmente los liberadores o afectivos, tan relacionados tradicionalmente con la literatura en su función social y formativa.

Nosotras apostamos por abrir caminos en el ideal de formación que podría recibir el alumnado de Primaria desde la educación literaria, reclamando que todos los valores de la educación integral cuenten con representación en esta área, sin reducir, por supuesto, la primacía que en ella se debe continuar otorgando a los valores estéticos.

Los puntos fuertes de este artículo son el desglose de los valores asociados al modelo axiológico defendido y su aplicación al currículo mínimo de Primaria, información que puede resultar útil al profesorado a la hora de seleccionar el material literario que podrían manejar en el aula. En este sentido, considerando las carencias del currículo evidenciadas en nuestro análisis, por un lado, y la literatura infantil como material formativo idóneo, por otro, 
instamos a considerar, seleccionar y evaluar muestras significativas procedentes de dicho campo que permitan la educación integral del alumnado.

Asimismo, dado que no parece conveniente que esta responsabilidad recaiga exclusivamente en el cuerpo de docentes, consideramos que el propio currículum debería instar de manera explícita a la selección de obras de la literatura infantil que incidan especialmente en los valores menos atendidos o ausentes en el bloque analizado del actual Real Decreto. Precisamente la categorización de un amplio corpus de LIJ bajo esta perspectiva es una línea de investigación que deja abierta este estudio.

Sirva, por tanto, esta contribución como estímulo para la reflexión acerca de cómo la literatura y el fomento del hábito lector contribuyen a la educación integral, precisamente al no enfocarse de manera supeditada a la educación en otros valores, pero también como propuesta de puntos clave que sirvan simultáneamente de estímulos para la reflexión y, sobre todo, para la mejora de próximas acciones normativas.

\section{REFERENCIAS BIBLIOGRÁFICAS}

Aparicio, I. (2016). La influencia de la educación literaria en la identidad de género: una propuesta educativa. Opción, 32(10), 36-53. Recuperado de https://www.redalyc.org/html/310/31048901003/

Bardin, L. (2002). El análisis de contenido (3. a ed.). Madrid: Akal.

Bassa i Martín, R. M. (2003-2004). Libros y lecturas para jóvenes. La transmisión de valores a través de la literatura infantil y juvenil: el caso de la LIJ catalana 1939-1985. Historia de la Educación. Revista Interuniversitaria, 22-23, 167-193.

Borda, M. I. (2000). Ideología y valores éticos en la literatura infantil y juvenil española actual. Puertas a la lectura, $3(\mathrm{~S}), 28-33$.

Cerrillo, P. C. (1996). Literatura infantil, hábitos lectores y educación de valores. En S. Yubero y E. Larrañaga (Coords.), El desafío de la educación social (pp. 213-220). Cuenca: Universidad de Castilla-La Mancha.

Cerrillo, P. C., y Yubero S. (1996). Literatura infantil y educación de valores: una selección bibliográfica. En S. Yubero y E. Larrañaga (Coords.), El desafío de la educación social (pp. 221-234). Cuenca: Universidad de CastillaLa Mancha.

Etxaniz, X. (2008). Investigación en torno a la literatura infantil y juvenil. Revista de Psicodidáctica, 13(2), 97-109.

Fraga de Azevedo, F. J. (2005). Ética y estética en la literatura de recepción infantil. Ocnos. Revista de Estudios sobre Lectura, 1, 7-18.

Gervilla, E. (2000). Un modelo axiológico de educación integral. Revista Española de Pedagogía, 215(1), 39-58.

Gervilla, E. (2008). Buscando valores: análisis de contenido axiológico y modelo de educación integral. En J. M. Touriñán (Dir.), Educación en valores, sociedad civil y desarrollo cívico (pp. 56-73). La Coruña: Netbiblio.

González, J. y Nieto, S. (2001). Investigación y didáctica sobre los valores en la literatura infantil: Un ejemplo...La 
isla del tesoro. Enseñanza es Teaching: Revista Interuniversitaria de Didáctica, 19, 141-164.

Huertas, R. M. (2006). Literatura infantil: el cuento y su valor educativo. En E. Mirafloresy J. Quintanal (Coords.), Educación infantil: orientaciones y recursos metodológicos para una enseñanza de calidad (pp. 353-376). Madrid: CCS.

Llorens, R. F. (2000). Literatura infantil y valores. Puertas a la Lectura, 9-10(S), 75-78.

López-García-Torres, R. (2012). Valores de la Ley de Educación de Andalucía y sus implicaciones educativas. Granada: Editorial Universidad de Granada. Recuperado de http://hera.ugr.es/tesisugr/20759800.pdf

López-García-Torres, R. y Saneleuterio, E. (2015). Los valores estéticos y literarios en la Ley de Educación de Andalucía: estudio para su revisión. En AIDIPE (Ed.), Investigar con y para la sociedad. Vol. 1 (pp. 315-325). Madrid: Bubok Publishing S.L. Recuperado de https://dialnet.unirioja.es/servlet/libro? codigo=572339

López-García-Torres, R. y Saneleuterio, E. (2019). El carácter fundamentante de los valores en la educación. Propuesta de un modelo axiológico de educación integral. Quién. Revista de Filosofía Personalista, 9, 39-61.

M. F. R. (2015). Los docentes reivindican las áreas artísticas ante la LOMCE. Diario de Mallorca, 13 de febrero [en línea]. Recuperado de http://www.diariodemallorca.es/mallorca/2015/02/13/docentes-reivindican-areasartisticas-lomce/999070.html

Martí, M. (2004). Los valores en la Literatura Infantil y Juvenil. Primeras Noticias. Revista de Literatura, 201, 19-20.

Ministerio de Educación, Cultura y Deporte (2014). Real Decreto 126/2014, de 28 de febrero, por el que se establece el currículo básico de la Educación Primaria. BOE, 52, 1 de marzo, 19349-19420.

Nieto, S. y González, J. (2002). Los valores en la literatura infantil: estudio empírico, técnicas y procedimientos de análisis. Valladolid: Aral.

Rodríguez, B. y Gutiérrez, R. (2013). Literatura y educación en valores: el problema de la utilización de la obra literaria como instrumento. Didácticas Específicas, 8, 30-44.

Sánchez, L. (1995). Literatura y lenguaje literario. Barcelona: Paidós.

Saneleuterio, E. y López-García-Torres, R. (2017). Los valores literarios en las enseñanzas de régimen general. El caso de la Ley de Educación de Andalucía. Profesorado. Revista de Currículum y Formación del Profesorado, 21(4), 337-358. Recuperado de https://recyt.fecyt.es/index.php/profesorado/article/view/62509/pdf_33

Sousa, S. (2008). Valores y formación en la literatura infantil y juvenil actual. Espéculo: Revista de Estudios Literarios, 39. Recuperado de http://webs.ucm.es/info/especulo/numero39/liteinfa.html

Tejerina, M. I. y Echevarría, E. (2007). Formación del lector y educación en valores: estética y ética de la literatura. En P. C. Cerrillo, C. Cañamares y C. Sánchez (Coords.), Literatura infantil. Nuevas lecturas y nuevos lectores (pp. 269-278). Cuenca: Universidad de Castilla-La Mancha. 


\section{INFORMACIÓN SOBRE LAS AUTORAS}

Elia Saneluterio. Es licenciada en Filología Hispánica y doctora europea en Literatura Española. Desde 2012 es profesora de Magisterio e investigadora en el Departament de Didàctica de la Llengua i la Literatura de la Universitat de València. Ha recibido varios premios por su labor académica y profesional, ha participado en más de treinta congresos de sus especialidades y es autora de numerosos capítulos de libros, artículos de investigación y monografías. Entre sus líneas de investigación actuales destaca su interés por las manifestaciones de la ficción infantil, los valores de la educación, la coeducación y las cuestiones de género, así como su repercusión en contextos académicos y culturales.

\section{elia.saneleuterio@uv.es.}

Rocío López García-Torres. Es licenciada en Psicología y doctora en Ciencias de la Educación por la Universidad de Granada. Desde 2010 es profesora de la Universidad CEU Cardenal Herrera, donde imparte materias del Área de Psicología Evolutiva y de la Educación. Su principal campo de estudio son los valores de la educación; también la coeducación, la violencia escolar y cualquier tema de relevancia educativa. Resultado de ello son los diversos artículos publicados en revistas científicas, las contribuciones a congresos de ámbito nacional e internacional y los capítulos de libros en los que aparece como autora.

$\bowtie$ rocio.lopez@uchceu.es 